\title{
Integrating Behavioral Health With Medical Services: Lessons From HIV Care
}

\author{
Eugene W. Farber, Kimya E. Hodari, \\ Veronica J. Motley, Blessing E. Pereira, \\ Magdalene Yonker, and Sanjay Sharma \\ Emory University School of Medicine
}

\author{
Peter E. Campos \\ PECDOC Research and Evaluation Services, Decatur, Georgia
}

\begin{abstract}
Recent trends have emerged that focus on developing strategies for integrating behavioral health services into medical care settings, prompting heightened interest in articulating specific models for integrated care. The biopsychosocial complexity of HIV disease has resulted in efforts to integrate HIV-related mental health services with medical treatment as part of an overarching continuum of care concept. Lessons learned from these efforts have the potential to contribute significantly to informing current deliberations regarding how to go about integrating mental health care with medical care in general medical settings. With the hope of contributing to the professional dialogue in this area, this article presents a detailed account of a comprehensive HIV mental health services program that is integrated with HIV/AIDS medical care. The program provides services for a traditionally underserved client population in a community-based urban setting. After elucidating the conceptual foundations of the program, key strategies for program implementation are described in detail. Finally, drawing upon the experiences and lessons learned from implementation of this integrated HIV mental health services model, the article concludes with an outline of specific implications for the evolving discussion regarding how best to integrate behavioral health services into general medical care settings.
\end{abstract}

Keywords: HIV, AIDS, mental health services, services integration, health psychology

Recent years have witnessed an increasing focus on articulating ways to integrate behavioral health with primary care services (e.g., Auxier, Farley, \& Seifert, 2011). Integrated care models based conceptually on a whole person perspective of health and illness (e.g., the patient centered medical home) are garnering attention both because of their clinical promise and their potential to contribute to the cost effectiveness of primary care (Runyon, 2011). As the trend toward integrated care approaches continues to unfold, there is a pressing need to elaborate specific frameworks of care. Lessons learned from the HIV/AIDS health care arena can inform these efforts, since the biopsychosocial complexity of HIV disease has long challenged HIV/AIDS providers to conceptualize care delivery in the context of a coordinated continuum of health services (Treisman \& Angelino, 2004; Winiarski, 1997). As such, this article describes a model of integrated HIV mental health service delivery designed specifically for underserved communi-
This article was published Online First June 18, 2012.

EUGENE W. FARBER received his $\mathrm{PhD}$ in clinical psychology from Georgia State University. He is an associate professor of Psychiatry and Behavioral Sciences, Emory University School of Medicine. His professional and research interests focus on psychotherapy, HIV-related psychological adaptation, and HIV mental health services.

KIMYA E. HODARI received her MA degree in counseling psychology from Argosy University, Atlanta. She is a project coordinator for HIV mental health services in the Department of Psychiatry and Behavioral Sciences, Emory University School of Medicine. Her clinical interests are in HIVrelated counseling and psychotherapy.

VERONICA J. MOTLEY received her EdD degree in counselor education and supervision from Argosy University, Atlanta. She is a mental health clinician in the Department of Psychiatry and Behavioral Sciences, Emory University School of Medicine. Her professional interests include HIVrelated clinical service provision and counselor training and supervision. Blessing E. PEREIRA received her MA degree in counseling psychology from Argosy University, Atlanta. She is a mental health clinician in the Department of Psychiatry and Behavioral Sciences, Emory University School of Medicine. Her clinical interests focus on mental health intervention for individuals living with chronic illness, including HIV disease.
MAGDALENE YONKER received her BA degree in psychology from Clark Atlanta University. She is a case manager in the Department of Psychiatry and Behavioral Sciences, Emory University School of Medicine. She specializes in case management services for individuals with co-occurring mental health concerns and chronic illness, with a focus on HIV/AIDS. SANJAY SHARMa received his MD degree from Emory University and completed a psychiatry residency in the Department of Psychiatry and Behavioral Sciences, Emory University School of Medicine, where he currently is an assistant professor. His clinical and research interests are in psychosomatic medicine and HIV-related neuropsychiatry.

Peter E. CAmpos received his PhD degree in clinical psychology from the University of Hawaii at Manoa. He is an independent applied research consultant based in Decatur, Georgia. His areas of research interest include behavioral medicine and health psychology, with a particular focus on HIV/AIDS mental health services and prevention.

THIS WORK WAS SUPPORTED by a grant from the Substance Abuse and Mental Health Services Administration (SAMHSA grant no. 5U79SM057701).

CORRESPONDENCE CONCERNING THIS ARTICLE should be addressed to Eugene W. Farber, Department of Psychiatry and Behavioral Sciences, Emory University School of Medicine, Grady Infectious Disease Program, 341 Ponce de Leon Avenue, Atlanta, GA 30308. E-mail: efarber@emory.edu 
ties disproportionately affected by HIV/AIDS. The overarching intent is to contribute to the evolving dialogue on integration of behavioral health services with primary care.

\section{Rationale for Integrating Mental Health Services in HIV/AIDS Care}

The emotional toll of HIV/AIDS is reflected in the remarkably high prevalence of co-occurring psychological disorders among those living with the illness. For instance, in one study nearly $50 \%$ of a nationally representative sample of individuals living with HIV screened positive for a psychiatric disorder (Bing et al., 2001). Similarly, past-year and past-month prevalence of a psychological disorder diagnosed using criteria from the Diagnostic and Statistical Manual of Mental Disorders, Fourth edition (DSMIV; American Psychiatric Association, 1994) in an HIV primary care sample was $50 \%$ and $33 \%$, respectively, with $50 \%$ of those with past-year and $40 \%$ of those with past-month DSM-IV diagnoses meeting criteria for more than one diagnosis (Gaynes, Pence, Eron, \& Miller, 2008). When not addressed as part of comprehensive HIV clinical management, psychological complications can compromise adherence to HIV treatment (Gonzales, Batchelder, Psaros, \& Safren, 2011), contribute to missed medical appointments (Rajabiun et al., 2007), and adversely affect HIV disease progression, including immunological functioning (Leserman, 2008). Clinically significant mental health concerns such as depression also may interfere with uptake of HIV behavioral sexual risk prevention interventions (Safren, Reisner, Herrick, Mimiaga, \& Stall, 2010). As such, HIV-related behavioral health interventions have a key role to play in comprehensive HIV/AIDS care, with the extant evidence base demonstrating their benefits for reducing psychological symptoms and enhancing emotional wellbeing, improving medication adherence, and reducing HIV transmission risk behavior (Safren et al., 2009; Scott-Sheldon, Kalichman, Carey, \& Fielder, 2008; Sikkema et al., 2010; Treisman \& Angelino, 2004).

The national HIV/AIDS strategy (NHAS; Millett et al., 2010), released in 2010, includes a goal to increase service access and improve health outcomes and action steps involving care coordination and collaboration among care providers. In line with this goal is the elaboration of integrated HIV health service models that include mental health programs (for review, see Soto, Bell, \& Pillen, 2004). Given the disproportionate impact of the United States HIV epidemic in communities of color, with the greatest burden among African Americans (Centers for Disease Control, 2011; Lansky, Brooks, DiNenno, Heffelfinger, Hall, \& Mermin, 2010), ensuring that clinical models are relevant to the service needs of these communities is especially important.

Although an integrated care delivery approach has been recommended for underserved individuals from diverse sociodemographic and cultural backgrounds living with a range of chronic health conditions, including HIV disease (Petersen, Hutchings, Shrader, \& Brake, 2011), the published literature includes only a handful of detailed model descriptions for implementing mental health programs within HIV medical care settings. For example, Feingold and Slammon (1993) described a hospital-based linkage model involving a family focused service framework with varying levels of HIV mental health intervention. Stepleman, Hann, Santos, and House (2006) out- lined a psychological consultation model for HIV medical care settings, and Dodds et al. (2000) reported a standardized method for assessing mental health needs and services planning for women in HIV primary care. With the aim of contributing further to the professional dialogue in this area, and consistent with NHAS priorities, this article describes the Alafia Project, a program that offers a comprehensive spectrum of mental health treatment and wellness services provided by a multidisciplinary clinical team. Funded by a Substance Abuse and Mental Health Services Administration (SAMHSA) HIV mental health services grant, the program is tailored for individuals from underserved communities, with a particular focus on an African American clientele. After articulating the conceptual foundations of the program, the scope of services offered and key program implementation strategies are described. The article concludes with reflections on implementation challenges, lessons learned, and general implications for efforts to integrate behavioral health services within medical care settings.

\section{Conceptual Foundations of the Alafia Project Service Approach}

\section{Biopsychosocial Perspective}

The biopsychosocial perspective frames patterns of mutual influence across physical/medical, psychological/emotional, social, and psycho-spiritual domains of functioning (Treisman \& Angelino, 2004; Winiarski, 1997). Anchored by general systems theory, the biopsychosocial framework highlights relationships among ". . .all the levels of organization pertinent to health and disease, from subatomic particles through molecules, cells, tissues, organs, organ systems, the person, the family, the community, the culture, and ultimately the biosphere" (Engel, 1978, p. 175). Accordingly, biomedical events (e.g., decline in HIV disease immune markers, opportunistic infections, neurocognitive changes, medication side effects) can precipitate psychological distress or exacerbate preexisting psychological susceptibilities while psychological and/or neuropsychiatric conditions may affect HIV disease progression and mortality (Treisman \& Angelino, 2004). Psychological factors (e.g., degree of emotional well-being, history of or current psychological disorder, ways of coping, attitudes, health beliefs) influence patterns of HIV-associated emotional adaptation. Psychosocial issues (e.g., limited material and financial resources, stigma, low social support) may contribute to the stress burden of living with HIV and influence access to health-related services as well as medical care retention and adherence. Also, spiritual beliefs can influence HIV-related meaning construction.

\section{Client-Centered Care Framework}

Having gained increasing traction in recent years as a framework for health care delivery, a client-centered care orientation incorporates client perspectives on problems and their solutions, considers client values, is collaborative, and encourages the elaboration of a shared understanding between clinician and client of problems and plans for treatment (McCormack et al., 2011). The client-centered care framework injects flexibility into the Alafia Project's service delivery system and encourages clinicians to engage their clients in a collaborative process of 
decision-making about their care. Consistent with the current evidence base demonstrating the importance of collaboration and consensus building in the treatment process (e.g., Tryon \& Winograd, 2011), clinicians routinely ask clients for input and feedback about their experiences of treatment and their appraisals of its effectiveness and relevance in addressing their concerns.

\section{Cultural Intersectionality}

The rich diversity of sociodemographic backgrounds and life experiences represented among individuals living with HIV/ AIDS, along with the spectrum of beliefs, norms, and values that flow from them, cannot be encapsulated in a singular way. As such, the concept of intersectionality (Brown, 2009) guides the Alafia Project's efforts to provide culturally competent care. Intersectionality refers to the ways in which the multiplicity of sociocultural dimensions that comprise a given individual's personal background (e.g., age, gender, race, ethnicity, sexual orientation, national/regional origins, spiritual/religious background, socioeconomic status) converge uniquely to inform that person's social and personal identity and experience. Brown (2009) characterizes intersectionality as the idea that ". . .each of us is more than the most obvious component of our identity and that these mixtures of aspects of self occur in a myriad of ways" (pp. 344-345). Intersectionality underscores the importance of striving to understand how a client's perceptions, beliefs, attitudes, emotional experience, and behavior are embedded within a richly textured coalescence of multiple diversity characteristics. It also orients clinicians to be reflective about how their own cultural intersectionality informs their clinical expectations, conceptualizations, relational stances, and intervention approaches in working with clients.

\section{The Scope and Flow of Service Delivery}

Anchored by these conceptual foundations, the scope and flow of service delivery is conceptualized broadly along a pathway that begins with client engagement and moves through comprehensive biopsychosocial assessment, services planning, and services types (see Figure 1). Clients may access services via self-referral or by referral from medical providers. In the assessment interview, current problems and concerns are explored along with personal strengths and potentialities, health beliefs, developmental history, mental health treatment history, current and past substance use, HIV and general health history, medical adherence, HIV risk behavior patterns, psychosocial background, and cultural context. Based on assessment findings, the client and clinician collaboratively develop an individualized services plan. The service approach is deliberately flexible so that the optimal type, frequency, and intensity of services can be selected based on clinical need, comfort level, and readiness for participation. As detailed in Figure 1, clinical programming is organized along two tracks. The first track encompasses treatment services for clinically significant psychological concerns or disorders and AIDS-related neurocognitive complications. The second track is comprised of wellness services designed to enhance resilience, coping, and adaptation. Clients can participate in services within one or both of these tracks serially or in combination. For example, a client with depression and housing instability may be offered a combination of individual psychotherapy, medication management, and housing-related case management on the treatment track, and a

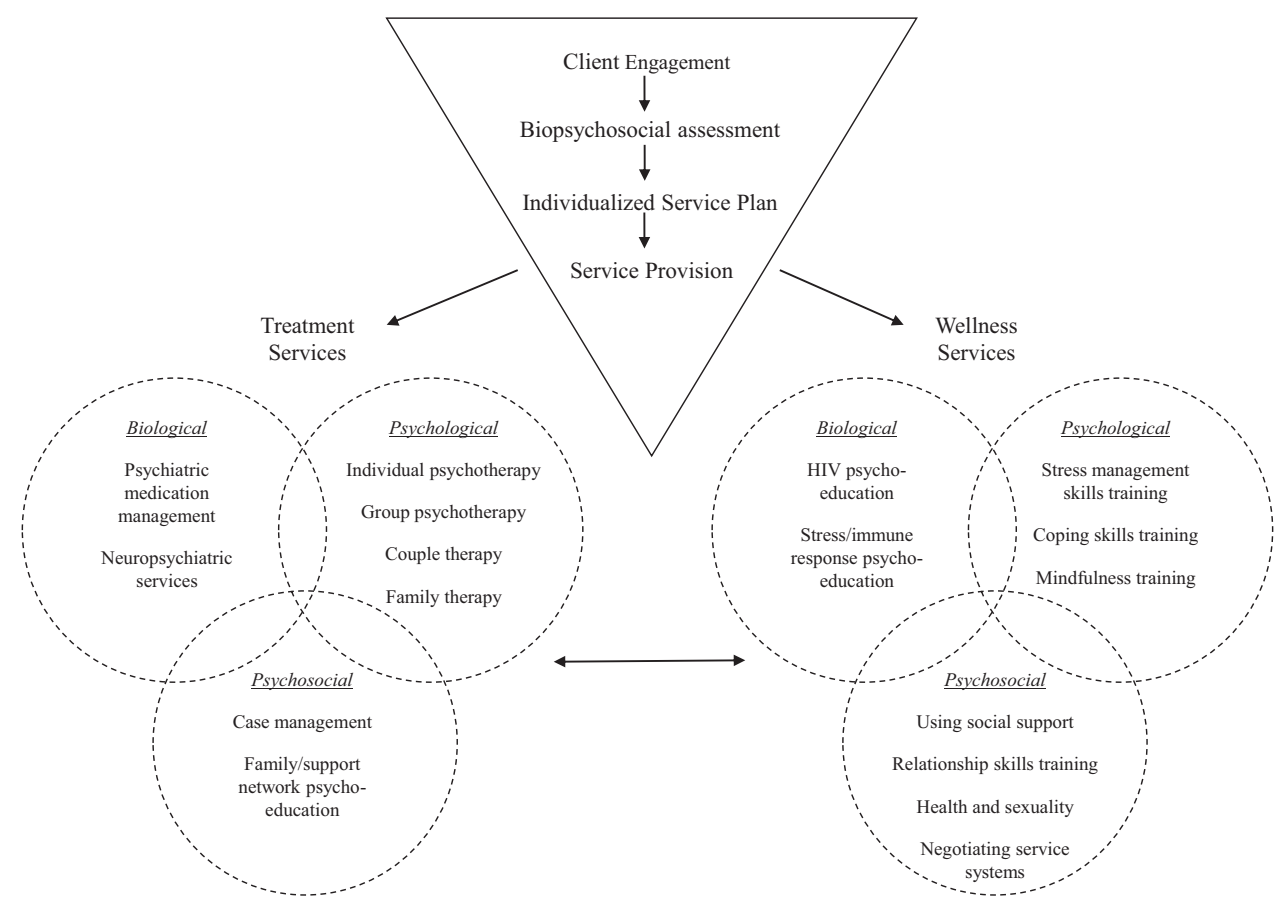

Figure 1. Scope and flow of services. 
mindfulness training group on the wellness track. Co-occurring substance use is addressed in the context of Alafia Project service delivery, although some clients may also require referral to outside community partner agencies for intensive substance use intervention.

\section{Key Programmatic Strategies}

\section{Services Integration}

Based in an academic health sciences center, the Alafia Project is colocated (i.e., clients can receive services on-site where they receive their medical services) within three HIV medical care settings in an urban HIV epicenter in the southeastern region of the United States that serve a sociodemographically diverse clientele with traditionally limited access to health care services. These settings include two ambulatory HIV primary care clinics and a general hospital inpatient HIV/AIDS medical specialty service. The Alafia Project team is comprised of a psychologist, psychiatrist, three master's prepared licensed mental health clinicians, and a mental health case manager. Diverse intersecting sociodemographic and cultural identities are represented among the program staff. As Project Director, the psychologist provides administrative oversight of all activities and services. The lead mental health clinician serves as Clinical Director, overseeing and coordinating the activities of the clinical team. Both the Project Director and Clinical Director work with administrative and clinical leadership at each service site to address logistical issues and ensure continuity of service delivery.

Some program elements are not just colocated but embedded seamlessly with medical services, with mental health providers fully integrated as members of transdisciplinary clinical teams. For instance, an Alafia Project clinician works alongside medical providers as a member of an HIV/Oncology specialty clinic team, provides real-time consultation on mental health aspects of care, routinely meets with new clients as part of the clinic enrollment protocol, and coordinates ongoing mental health visits with medical and/or chemotherapy visits.

\section{Whole Person Orientation to Service Provision}

In the West African Yoruba culture, Alafia is a term for wellbeing that combines the ideals of physical health, peace of mind, strength, prosperity, harmonious relationships, and wisdom. This meaning reflects both the Alafia Project's whole person framework for conceptualizing health and well-being and its aspirational goal of maximizing the adaptive potential of individuals living with HIV disease. Accordingly, the client is understood as a biopsychosocial unity, with a focus on understanding the client's psychological challenges and resources as a totality to ensure full appreciation of both problems and potentialities.

Evolving approaches to HIV care highlight the importance of cultivating personal agency and empowering clients to be informed and active in the self-management of their illness (Swendeman, Ingram, \& Rotheram-Borus, 2009). Consistent with this framework, the Alafia Project's service delivery approach combines a traditional psychotherapy focus on reducing symptoms and problems with an emphasis on facilitating active coping and framing personal challenge as an opportunity for psychological growth.
The program's evidence-based HIV specific mental health wellness groups, including stress management groups (Antoni, Ironson, \& Schneiderman, 2007), also promote adaptive coping and self care.

Informed broadly by a solution-focused model (Greene et al., 2006), the Alafia Project's case management approach focuses on client strengths, skills, and personal resources. Accordingly, the case manager invites the client to view problems as "teachable moments" for illuminating strategies for effectively solving problems, making decisions, advocating for self, and navigating complex systems. Aiming to help clients experience increasing levels of competence, confidence, and autonomy, the case manager seeks an optimal balance between providing emotional and instrumental support and promoting the client's sense of agency in formulating solutions to address social services challenges.

\section{Cultivating Culturally Competent Service Delivery}

Guided by the program's foundational orientation to cultural intersectionality (Brown, 2009), providing culturally competent services is viewed as an ongoing process nurtured by a commitment to a clinical attitude of openness, curiosity, and flexibility with respect to similarities and differences in the context of providing care. In seeking to offer services that are both relevant and responsive to the needs and concerns of a richly diverse clientele, priority is placed on creating a culturally responsive and respectful care environment, including both the physical spaces in which care is delivered and the atmosphere of openness and receptivity cultivated by the program's staff. The Alafia Project's foundational emphasis on a collaborative client-centered service delivery approach, along with a focus on supporting client strengths also are key programmatic elements aimed at cultivating culturally competent service delivery. Conceptually, these characteristics are broadly consistent with the framework of the evidence-based Patient-Centered Culturally Sensitive Health Care model (Tucker, Marsiske, Rice, Nielson, \& Herman, 2011).

Within a cultural intersectionality framework clinical work involves a dynamic process of inquiry focusing on both similarity and difference in the service of the client's clinical needs and concerns. Concretely, this process requires the clinician substantively to consider the client's psychological life in the context of the intersection of a range of sociodemographic dimensions (e.g., age, gender, race, ethnicity, nationality, sexual orientation, socioeconomic status, language, literacy, and disability). For example, in working with an African American woman with limited financial means referred by a medical provider for behavior characterized as problematically "defensive and uncompromising," the mental health clinician may seek to explore the meaning of these behaviors in the context of multiple intersecting strands of the client's identity in an effort to understand their significance from the client's point of reference (Brown, 2009). One such strand may relate to her role expectations and experiences as a caregiver of an extended family. A second strand may involve her prior experiences with disparities in care and health care system unresponsiveness. A third strand may pertain to the reality that, owing to her socioeconomic situation, this client has few choices for health services. Taking these three intersecting strands together, the clinician may conclude that the behavior that the medical provider perceives as problematic is, from the client's perspective, an 
expression of self-advocacy. While affirming the client's strengths and resilience in this regard, the clinician may also invite her to explore collaboratively alternative ways of perceiving and engaging with the medical provider that might better serve her wish to be her own advocate. Similarly, with the client's permission, the clinician might confer with the medical provider regarding alternative ways of understanding and responding to the client's behavior.

While men and women living with HIV share many common experiences, there are certain gender specific themes that are important to address in the context of clinical work. For instance, many women living with HIV describe challenges in balancing needs for self-care with familial, community, and societal expectations relative to traditional caregiver roles. Emotional concerns associated with these challenges are the focus of a time-limited Alafia Project women's group that explores self-esteem regulation, relationships, self-assertion, negotiating safer sex practices, spirituality, and HIV medication adherence using both cognitive and experiential exercises. Artistic expression also is utilized as a vehicle to facilitate self-exploration. An overarching aim of this group is to encourage participants, many of whom feel compelled to attend to the needs of others in their daily lives at considerable personal expense, to take time to focus on themselves in a reflective way. This group also provides an avenue for cultivating social support networks. Toward this end, periodic "alumni groups" are held, with participants from previous cohorts invited to attend.

Mental health stigma is a well-known challenge to engaging clients in mental health services, the impact of which may be influenced to varying degrees by sociocultural background (Brown, Conner et al., 2010; Ojeda \& McGuire, 2006). Some clients may experience seeking help and counsel from individuals outside of the usual support systems of family, close friends, and community networks as inconsistent with cultural norms or values, causing uneasiness and caution about accepting a mental health services referral. While the very presence of on-site mental health services in HIV medical care settings tends to have a normalizing impact for some clients, the Alafia Project offers specific programming aimed at reducing stigma-related barriers to care. A key example is the Wellness Series, an ongoing program of monthly drop-in presentations conducted by Alafia Project staff, medical providers, and/or community volunteer experts covering such topics as HIV health and self-care, art therapy, music therapy, laughter yoga, stress and relaxation, healthy cooking and nutrition, and accessing community social service resources. These sessions not only provide information and experiential learning opportunities but aim to reduce stigma by broadening client perceptions of the potential scope and relevance of mental health services for overall health and well-being.

Because of the stress burden of HIV stigma, along with its associations with reduced medical care access and adherence (Sayles, Wong, Kinsler, Martins, \& Cunningham, 2009) as well as decreased likelihood of retention in mental health care (Reece, 2003), the Alafia Project also has developed services targeting HIV stigma concerns. One example is the "Between Us" women's group, a weekly 10-session group focused on reducing stigmaassociated social isolation, increasing social connectedness and self-care, and, importantly, exploring disclosure as a positive coping strategy in a confidential and supportive environment. Because participants are apprehensive about disclosure of their HIV status, the group meets after regular clinic hours and group members can decide whether or not to identify themselves by name to fellow participants.

A key avenue for cultivating culturally competent service delivery involves inclusion of a consumer voice in program operations. This is accomplished via informal client feedback, ongoing monitoring of client perceptions of care in the context of service provision, consumer needs assessments and satisfaction surveys, and the activities of the Alafia Project's Consumer Advisory Board (CAB). Meeting monthly, the CAB is comprised of consumer volunteers who are both sociodemographically and clinically representative of the clients receiving services through the program. The $\mathrm{CAB}$ operates within a framework of bylaws formally established by the members that specify the requirements for $\mathrm{CAB}$ membership, meeting structure and procedures, and elections and terms of $\mathrm{CAB}$ officers (chair, cochair, secretary, and parliamentarian). The mental health case manager serves as the staff $\mathrm{CAB}$ liaison, providing logistical support and consultation when requested by the $\mathrm{CAB}$ to address challenges and concerns as they arise. Along with offering consumer perspectives on program design, planning, and service provision, CAB participants actively work to challenge client misconceptions about the purposes and uses of mental health services, often with the result that reluctant peers are increasingly open to utilizing these services. In addition to the substantial and wide-ranging contributions that the $\mathrm{CAB}$ makes to the program, $\mathrm{CAB}$ service frequently confers a sense of accomplishment and empowerment for consumer volunteers.

\section{Collaboration in Service Delivery}

Intrateam collaboration. While each client receiving Alafia Project services has a designated primary mental health provider, service delivery is fundamentally a collective effort. Professional role boundaries are "semipermeable" in that multiple staff may have varying levels of involvement in the care of a given client within the purview of their assigned clinical responsibilities and domains of professional expertise. For instance, a primary mental health provider may enlist colleagues to assist in managing a client crisis or an especially complex clinical situation. This approach contributes to a sense of shared clinical responsibility with clear benefits for clients, and permits the emotional impact of high intensity clinical circumstances to be absorbed by a mutually supportive clinical team rather than by a single clinician alone. Psychiatry services also are delivered collaboratively, with the psychiatrist and primary mental health provider frequently meeting together with the client. This approach confers the advantage of real-time care coordination between the primary mental health clinician and psychiatrist on behalf of the client, and also helps ease the apprehension some clients experience in meeting with a psychiatrist, particularly for the first time, thereby mitigating a potential barrier to care.

Collaboration with the medical team. Ongoing collaboration with medical staff as a part of routine client care is a key advantage of the colocation of mental health services in medical care settings. The presence of Alafia Project clinicians in the same clinics (and in some instances the same clinic hallways) as medical providers permits face-to-face care coordination in real time. As these collaborations have become a normative aspect of service delivery, even new medical providers quickly incorporate these 
collaborative strategies into their clinical routines. Significantly, even when not making formal referrals, medical providers frequently ask for input from their mental health colleagues about how best to communicate with their clients, address psychological concerns in the context of routine medical visits, or manage the challenges and stresses of working with particular clients or clinical situations.

\section{Prioritizing the Professional Development of the Mental Health Team}

Given the biopsychosocial complexity and intensity of HIVrelated clinical service delivery, professional development activities for mental health team members are incorporated into program operations both to enhance client services and mitigate staff burnout risk. These activities are voluntary and well attended by staff. Specifically, the Clinical Director offers weekly individual and group peer supervision for the clinicians and the case manager, and also holds weekly clinical team meetings with a focus both on clinical issues and staff attunement to work-related self-care. The Project Director convenes a monthly meeting where staff members discuss ongoing program operations and introduce ideas for innovations in the clinical service model. Team members also participate in annual retreats that afford opportunities to pause and reflect on program functioning as a whole and consider future directions.

Significantly, relationships among program staff have evolved over time to include shared participation in social activities outside of the work environment and in marking life milestones such as birthdays and weddings. Research suggests that shared professional activities, social activities, and life events all contribute meaningfully to sustaining health care teams (Brown, Lewis et al., 2010). Consistent with this finding, the Alafia Project team has enjoyed remarkable longevity, with high staff retention, cohesion, and a sense of shared ownership and mutual responsibility for program services, activities, and client care.

\section{Challenges in Program Implementation}

Alafia Project implementation has not been without logistical, systemic, and clinical challenges. Logistically, coordinating medical and mental health visits has necessitated flexibility in accommodating clients arriving late for mental health appointments when their medical visits run longer than expected. Since medical exam room areas are not designed with the privacy requirements for mental health service delivery in mind, finding the appropriate space to see clients is sometimes challenging. To address this problem, nearby offices proximal to medical exam room areas are used to ensure client privacy for mental health visits. Early efforts to offer in-hospital psychotherapy services for clients receiving inpatient AIDS-related medical care were significantly challenged by privacy concerns along with the fact that the majority of these clients were too acutely medically ill to make effective use of such services. As a result, the service emphasis shifted from providing in-hospital intervention to offering case management aimed at linking in-need clients to Alafia Project services upon hospital discharge.

Developing optimal transdisciplinary systems for care coordination has been an evolving process. For instance, early on mental health and medical staff had to learn how best to coordinate efforts so they did not work at cross purposes or unnecessarily duplicate services. As such, clarification of professional boundaries and role specification was a key early focus of planning meetings. In-services provided for medical staff by Alafia Project clinicians also helped to clarify roles, responsibilities, and expectations regarding mental health aspects of care. Also early on, Alafia Project clinicians struggled at times to keep up with the large number of requests by medical providers to see clients immediately for mental health evaluations. Over time, criteria for evaluating mental health acuity were developed to help medical providers distinguish clients with emergent immediate needs from those who could wait for a scheduled appointment. Overall, cultivating mutuality, good will, and a sense of shared responsibility in relationships with medical administrators and staff has been essential to programmatic success.

An ongoing clinical challenge pertains to engaging and retaining clients identified by medical providers as needing mental health services and yet reluctant to utilize these services because of stigma-related barriers. As noted earlier, one focus of the program's mental health wellness services has been to provide avenues for mental health services engagement that clients may experience as less stigmatizing than traditional service approaches. In some instances where clients are unwilling to engage directly in mental health treatment, Alafia Project clinicians work as consultants to guide medical providers in managing behavioral complications. An additional clinical challenge involves occasions where mental health-related client-system conflicts arise that generate significant barriers to care by creating staff splits among the medical team. Over time, a process has been developed for a systemic response directed by Alafia Project clinicians who facilitate a joint meeting with the client and medical staff to discuss concerns, clarify roles and expectations, and specify plans for managing conflicts as they arise.

\section{Conclusions: Lessons for Integrating Mental Health Services in Medical Settings}

HIV mental health specialty programs integrated within HIV medical settings have, in many respects, anticipated current trends in the health care system as a whole toward integrating mental health service delivery into medical care settings. As one example of such a program, the Alafia Project employs a conceptual and operational framework that is portable and can be applied readily to the development of integrated mental health programs within a broad range of medical care settings. With this in mind, and recognizing that there are innumerable potential pathways for developing integrated services approaches, several key lessons from the Alafia Project are highlighted with the hope that these might be helpful to future work in this area.

First, where possible, it is immensely valuable to articulate a core set of conceptual or theoretical principles from which can flow programmatic aims, structures, and intervention strategies. The biopsychosocial, client-centered, and cultural intersectionality concepts framed all aspects of the Alafia Project's implementation, including its design and clinical programs.

Second, achieving services integration requires active collaboration with key stakeholders in designing and implementing the program, including consumers and members of medical teams. 
Among the strengths of an integrated care approach is the opportunity to solicit multiple viewpoints regarding what is needed and valued in a particular setting. Consumer input is invaluable and mutually enriching both for the program and for the consumers themselves. Seeking input from medical team members also is critical to ensuring the success of service delivery and for building genuinely collaborative professional relationships on behalf of clients.

Third, it is important to broaden traditional concepts of the purposes and reach of mental health services in ways that reflect a whole person view of the individual as a biopsychosocial unity. From this perspective, while offering evidence-based interventions to treat psychological disorders remains important, programming also must focus on the role of mental health services relative to prevention of health problems and sustaining health and wellbeing.

Fourth, prioritizing a culturally responsive framework for services delivery is essential for providing integrated care. Careful attention should be paid to how program policies and procedures, the physical environment in which services are provided, clinical staffing, program brochures and literature, and the range and types of clinical services provided are responsive to the needs and concerns of an increasingly culturally diverse clientele.

Fifth, the quality of program services delivery hinges on the quality of relationships among staff members and their professional readiness for what is routinely experienced as high stress work. Actively and intentionally supporting staff relationships and professional development is crucial, as is taking time to cultivate relationships with transdisciplinary medical colleagues to ensure smooth collaboration in service delivery. As relationships between Alafia Project staff and their transdisciplinary medical colleagues have evolved, mutual appreciation for the activities and roles of each has deepened, including perceptions by medical providers of the value added by the presence of on-site mental health service providers.

Although beyond the scope and focus of this article, it is important to acknowledge challenges related to funding and program sustainability and their implications for the feasibility, staffing, size, and breadth of a mental health services program operating within a medical care setting. In recent years, SAMHSA has been a resource for grant support for developing programs focusing on primary and behavioral health integration. The emergence of patient-centered medical homes may yield additional opportunities for supporting this work. Where feasible, integrating mental health and medical services has significant advantages that can enhance a client's experience of care and improve health and well-being.

\section{References}

American Psychiatric Association. (1994). Diagnostic and Statistical Manual of Mental Disorders (4th ed.). Washington, DC: Author.

Antoni, M. H., Ironson, G., \& Schneiderman, N. (2007). Cognitivebehavioral stress management for individuals living with HIV: Facilitator guide. Oxford, UK: Oxford University Press.

Auxier, A., Tillman, F., \& Seifert, K. (2011). Establishing an integrated care practice in a community health center. Professional Psychology: Research and Practice, 42, 391-397. doi:10.1037/a0024982

Bing, E. G., Burnham, M. A., Longshore, D., Fleishman, J. A., Sherbourne, C. D., London, A. S., ... Shapiro, M. (2001). Psychiatric disorders and drug use among human immunodeficiency virus-infected adults in the United States. Archives of General Psychiatry, 58, 721-728. doi: 10.1001/archpsyc.58.8.721

Brown, C., Conner, K. O., Copeland, V. C., Grote, N., Beach, S., Battista, D., \& Reynolds, C. F., III. (2010). Depression stigma, race, and treatment seeking behavior and attitudes. Journal of Community Psychology, 38, 350-368. doi:10.1002/jcop.20368

Brown, J. B., Lewis, L., Ellis, K., Beckhoff, C., Stewart, M., Freeman, T., \& Kasperski, M. J. (2010). Sustaining primary health care teams: What is needed? Journal of Interprofessional Care, 24, 463-465. doi:10.3109/ 13561820903417608

Brown, L. S. (2009). Cultural competence: A new way of thinking about integration in therapy. Journal of Psychotherapy Integration, 19, 340353. doi: $10.1037 / \mathrm{a} 0017967$

Centers for Disease Control. (2011). HIV surveillance report, 2009 (p. 21). Atlanta, GA: Author

Dodds, S., Blaney, N. T., Nuehring, E. M., Blakley, T., Lizzotte, J. M., Potter, J. E., \& O'Sullivan, M. J. (2000). Integrating mental health services into primary care for HIV-infected pregnant and non-pregnant women: Whole Life - a theoretically derived model for clinical care and outcomes assessment. General Hospital Psychiatry, 22, 251-260. doi: 10.1016/S0163-8343(00)00090-6

Engel, G. L. (1978). The biopsychosocial model and the education of health professionals. Annals of the New York Academy of Sciences, 310, 169-187. doi:10.1111/j.1749-6632.1978.tb22070.x

Feingold, A., \& Slammon, W. R. (1993). A model integrating mental health and primary care services for families with HIV. General Hospital Psychiatry, 15, 290-300. doi:10.1016/0163-8343(93)90021-F

Gaynes, B. N., Pence, B. W., Eron, J. J., \& Miller, W. C. (2008). Prevalence and comorbidity of psychiatric diagnoses based on reference standard in an HIV+ patient population. Psychosomatic Medicine, 70, 505-511. doi:10.1097/PSY.0b013e31816aa0cc

Gonzales, J. S., Batchelder, A. W., Psaros, C., \& Safren, S. A. (2011). Depression and HIV/AIDS treatment nonadherence: A review and metaanalysis. Journal of Acquired Immune Deficiency Syndrome, 58, 181187.

Greene, G. J., Kondrat, D. C., Lee, M. Y., Clement, J., Siebert, H., Mentzer, R. A., \& Pinnell, S. R. (2006). A solution-focused approach to case management and recovery with consumers who have a severe mental disability. Families in Society, 87, 339-350.

Lansky, A., Brooks, J. T., DiNenno, E., Heffelfinger, J., Hall, H. I., \& Mermin, J. (2010). Epidemiology of HIV in the United States. Journal of Acquired Immune Deficiency Syndromes, 55, S64-S68. doi:10.1097/ QAI.0b013e3181fbbe15

Leserman, J. (2008). Role of depression, stress, and trauma in HIV disease progression. Psychosomatic Medicine, 70, 539-545. doi:10.1097/ PSY.0b013e3181777a5f

McCormack, L. A., Treiman, K., Rupert, D., Williams-Piehota, P., Nadler, E., Arora, N. K., . . Street, Jr., R. L. (2011). Measuring patient-centered communication in cancer care: A literature review and the development of a systematic approach. Social Science \& Medicine, 72, 1085-1095. doi:10.1016/j.socscimed.2011.01.020

Millett, G. A. Crowley, J. S., Koh, H., Valdiserri, R. O., Frieden, T., Dieffenbach, C. W., ... Fauci, A. S. (2010). A way forward: The national HIV/AIDS strategy and reducing HIV incidence in the United States. Journal of Acquired Immune Deficiency Syndrome, 55, S144S147. doi:10.1097/QAI.0b013e3181fbcb04

Ojeda, V. D., \& McGuire, T. G. (2006). Gender and racial/ethnic differences in use of outpatient mental health and substance use services by depressed adults. Psychiatric Quarterly, 77, 211-222. doi:10.1007/ s11126-006-9008-9

Petersen, S., Hutchings, P., Shrader, G., \& Brake, K. (2011). Integrating health care: The clear advantage for underserved and diverse populations. Psychological Services, 8, 69-81. doi:10.1037/a0023521 
Rajabiun, S., Mallinson, R. K., McCoy, K., Coleman, S., Drainoni, M., Rebholz, C., \& Holbert, T. (2007). "Getting me back on track": The role of outreach interventions in engaging and retaining people living with HIV/AIDS in medical care. AIDS Patient Care and STDs, 21 (Supplement 1), S20-S29. doi:10.1089/apc.2007.9990

Reece, M. (2003). HIV-related mental health care: Factors influencing dropout among low-income, HIV-positive individuals. AIDS Care, 15, 707-716. doi:10.1080/09540120310001595195

Runyon, C. N. (2011). Psychology can be indispensable to health care reform and the patient-centered medical home. Psychological Services, 8, 53-68. doi:10.1037/a0023454

Safren, S. A., O'Cleirigh, C., Tan, J. Y., Raminani, S. R., Reilly, L. C., Otto, M. W., \& Mayer, K. H. (2009). A randomized controlled trial of cognitive behavioral therapy for adherence and depression (CBT-AD) in HIV-infected individuals. Health Psychology, 28, 1-10. doi:10.1037/ a0012715

Safren, S. A., Reisner, S. L., Herrick, A., Mimiaga, M. J., \& Stall, R. D. (2010). Mental health and HIV risk in men who have sex with men. Journal of Acquired Immune Deficiency Syndrome, 55, S74-S77. doi: 10.1097/QAI.0b013e3181fbc939

Sayles, J. N., Wong, M. D., Kinsler, J. J., Martins, D., \& Cunningham, W. E. (2009). The association of stigma with self-reported access to medical care and antiretroviral therapy adherence in persons living with HIV/AIDS. Journal of General Internal Medicine, 24, 1101-1108. doi: 10.1007/s11606-009-1068-8

Scott-Sheldon, L. A. J., Kalichman, S. C., Carey, M. P., \& Fielder, R. L. (2008). Stress management interventions for HIV+ adults: A metaanalysis of randomized controlled trials, 1989 to 2006. Health Psychology, 27, 129-139. doi:10.1037/0278-6133.27.2.129

Sikkema, K. J., Watt, M. H., Drabkin, A. S., Meade, C. S., Hansen, N. B.,
\& Pence, B. W. (2010). Mental health treatment to reduce HIV transmission risk behavior: A positive prevention model. AIDS and Behavior, 14, 252-262. doi:10.1007/s10461-009-9650-y

Soto, T. A., Bell, J., \& Pillen, M. B. (2004). Literature on integrated HIV care: A review. AIDS Care, 16 (Supplement 1), S43-S55. doi:10.1080/ 09540120412331315295

Stepleman, L. M., Hann, G., Santos, M., \& House, A. S. (2006). Reaching underserved HIV-positive individuals by using patient-centered psychological consultation. Professional Psychology: Research and Practice, 37, 75-82. doi:10.1037/0735-7028.37.1.75

Swendeman, D., Ingram, B. L., \& Rotheram-Borus, M. J. (2009). Common elements in self-management of HIV and other chronic illnesses: An integrative framework. AIDS Care, 21, 1321-1334. doi:10.1080/ 09540120902803158

Treisman, G. J., \& Angelino, A. F. (2004). The psychiatry of AIDS: A guide to diagnosis and treatment. Baltimore, MD: The Johns Hopkins University Press.

Tryon, G. S., \& Winograd, G. (2011). Goal consensus and collaboration. Psychotherapy, 48, 50-57. doi:10.1037/a0022061

Tucker, C. M., Marsiske, M., Rice, K. G., Nielson, J. J., \& Herman, K. (2011). Patient-centered culturally sensitive health care: Model testing and refinement. Health Psychology, 30, 342-350. doi:10.1037/a0022967

Winiarski, M. G. (1997). Understanding HIV/AIDS using the biopsychosocial/spiritual model. In M. G. Winiarski (Ed.), HIV mental health for the 21st century (pp. 3-22). New York, NY: New York University Press.

Received December 19, 2011

Revision received March 22, 2012 Accepted April 27, 2012 